\title{
Physicalism, Introspection, and Psychophysics: The Carnap/Duncker Exchange
}

\author{
Uljana Feest
}

Leibniz Universität Hannover

October 2014

In 1932, Rudolf Carnap published his article "Psychology in a Physical Language." The article prompted a critical response by the Gestalt psychologist Karl Duncker. The exchange is marked by mutual lack of comprehension. In this paper I will provide a contextualized explication of the exchange. I will show that Carnap's physicalism was deeply rooted in the psychophysical tradition that also informed Gestalt psychological research. By failing to acknowledge this, Carnap missed out on the possibility to enter into a serious debate and to forge an alliance with a likeminded psychologist at the time. I conclude by suggesting that the kind of physicalism practiced by Gestalt psychologists deserves to be taken seriously by current philosophy of psychology.

In the early 1930s, Rudolf Carnap laid down his project of overcoming metaphysics by means of linguistic analysis (Carnap 1931a) and specified a universal (physical) language as the language of choice (Carnap 1931b). It is well known that Carnap's 1931b article gave rise to what is often referred to as the "protocol-language debate" within the Vienna Circle (Neurath 1932; Carnap 1932b). While there is some impressive historical and philosophical scholarship about this debate (e.g., Uebel 2007), one strand of it has not received much attention, namely the ways in which Carnap's views about the physicalizability of protocol sentences were related to research in experimental psychology at the time. This is especially surprising given the fact that Carnap, in his article "Psychology in a Physical Language" (1932a) attempted to spell out the implications of this view for psychology. This article was met by a critical response by the psychologist Karl Duncker (1932), which in turn prompted a reply from Carnap (1932c). The exchange is characterized by a surprising degree of mutual incomprehension, with Duncker suggesting that Carnap's critique of (introspective) psychology was attacking a strawman and Carnap saying that Duncker had completely missed his point.

In this article I will explicate and contextualize the exchange between Carnap and Duncker. I will shed some light on the reasons why the two talked past each other and I will show that Duncker did put his finger on the fact that (1) Carnap's position failed to address scientific practice, and 
that (2) Carnap did indeed attack several strawmen. I will lay out that Carnap's turn to a physical language was motivated by his aim to provide an objective foundation for protocol sentences (section 2), and argue that the way in which Carnap executed his project of physicalizing protocol-sentences was deeply informed by psychophysics (section 3). I will then (in section 4) turn to Carnap's 1932a article "Psychology in a Physical Language," where he claims to be addressing the implications of his views for psychology. Section 5 details Duncker's response and Carnap's answer. Finally, in section 6, I will draw out two underlying issues in this debate, i.e., (a) the status of introspection in psychological research, and (b) the question what (if any) metaphysical presuppositions were made by psychophysical research.

\section{Overcoming Metaphysics and the Problem of Experience}

In his famous article "Overcoming Metaphysics" Carnap laid out the very lean conception of philosophy characteristic of the Vienna Circle (Carnap 1931a). According to it, philosophy was neither to engage in metaphysical speculations about age-old topics, nor in naturalistic treatments of them. Rather, it was essentially reduced to providing meta-analyses of existing discourses in order to clean them of "pseudo-sentences;" sentences that look grammatically like sentences, but are in fact meaningless. The method of choice (logical analysis of language) was to proceed by translating every sentence that is formulated in the so-called "material mode of speech" into a sentence in the "formal mode of speech" (a sentence about a sentence). This method was to reveal whether a given statement was logically consistent and empirically meaningful.

In response to the question of what it takes for a statement to be empirically meaningful, Carnap introduced a version of the well-known verificationist criterion of meaning that we still associate closely with the doctrine of logical positivism. According to it a word $\boldsymbol{a}$ is meaningful only if (1) empirical indicators for a are known, (2) it is known what protocol sentences the sentence $\mathrm{S}(\boldsymbol{a})$ can be derived from, and (3) the path towards verifying S(a) is known (Carnap 1931a, 224). Carnap's verificationist semantics for words emphasized the empirical truth conditions for sentences in which the words occur. These empirical truth conditions were provided by "observational" or "protocol-sentences" and he stated that while there was to date no agreement 
about the form or content of such sentences, they were commonly thought to refer to something that is "given" (Carnap 1931a, 222).

This raised the question of what were criteria of meaningfulness of protocol sentences themselves? Was their meaningfulness ensured by a primitive notion of the "given," i.e., by the subjective experience that is - in the material mode - reported by protocol sentences? Or was there a more 'public' way of stating the truth conditions for protocol sentences? It is this question that Carnap addressed in his "Physical Language as the Universal Language of Science" (Carnap 1931b), where he argued that both protocol sentences and "system sentences" (i.e., sentences capable of being derived from, and verified, by protocol sentences) are part of an overarching language: the universal language of science. By the requirement of universality, Carnap meant that such a language "can describe every state of affairs" (Carnap 1963 [1932a] 400). But in addition he argued that such a language should also be intersubjective, i.e., it should be usable by everybody in the same way. It is in this second respect that Carnap's aim in this work differed from the one proposed in his 1928 Aufbau, where he had wanted the universal language to be that of subjective experience. It was because of the requirement of intersubjectivity that Carnap turned to the physical language as the universal language (see Uebel 2007). ${ }^{1}$

Carnap's thesis that (seemingly subjective) protocol sentences were translatable into the (intersubjective) language of physics was ostensibly part of a larger thesis, namely that all sentences are translatable into sentences of the physical language. Surprisingly, Carnap did not present an argument for this larger thesis, except to suggest that its truth was obvious, at least in the case of "the inorganic sciences" (chemistry, geology, astronomy) and even biology since they were (in the material mode) dealing with physical objects. However, since his main target was the physicalizability of protocol sentences, a separate argument was required, and he attempted to provide one in sections 4 of his "Physical Language."

\footnotetext{
${ }^{1}$ In his "Physical Language" article he still maintained that protocol-sentences were the most basic sentences of science that could not themselves be doubted (438), but in response to Neurath's critique, he revised this position to say that any scientific sentence within a physicalist system could function as a protocol sentence ("Über Protokollsätze" 224) and no sentence could function as an ultimate epistemic basis (225).
} 


\section{The Psychophysical Underpinnings of Physicalized Protocol Sentences}

Carnap's argument for the translatability of protocol sentences into physical sentences took the form of an empirical claim: He posited that it is possible to find a quantitative equivalent for every qualitative (protocol) sentence, and he noted that this is not a logical necessity but simply a contingent empirical fact, such that there is a functional dependency between protocol sentences and physical sentences. (In the material mode, it is an empirical fact about the way in which our experience is structured in relation to the physical world). From this, Carnap derived the claim that it is possible to find a corresponding physical sentence for every protocol sentence, a fact that provides the basis of the very possibility of practicing an intersubjective physics.

It is clear that Carnap expected the physical sentences in question to be sentences about the brain, but realized that neuroscience at the time did not deliver sentences that directly corresponded to sentences about experiences. For this reason, he decided to describe the relevant brain states in terms of behavioural dispositions, specifically, the disposition to display particular behaviours in response to particular stimuli. This was made explicit in his subsequent "Psychology in a Physical Language," but is already apparent in the way he explains his position in his $1932 \mathrm{~b}$ paper. For example, he states that it is possible to establish empirical correlations between the qualitative statements of protocol sentences and the quantitative determination (in a physical language) of the conditions under which they are uttered. For example, when examining color vision, he argued, one has to vary "the physical conditions (e.g., the combination of various frequencies of oscillations) and discover the conditions to which $\mathrm{S}$ reacts with the protocol statements containing the qualitative terms in question" (Carnap 1963 [1932]; 408). Then he states "The discovery of the set of these physical terms corresponding to a definite qualitative term will be called the "physicalizing" ... of this qualitative term" (ibid.).

Unfortunately, Carnap does not provide a reference for this, but it is striking that there was in fact a research program that did just what Carnap was describing: i.e., vary physical stimulus conditions and measure responses. This research program, psychophysics, had famously been founded by Fechner (1860) and continues to be active until today (Heidelberger 2004). At the time of Carnap's work in the 1930s, famous proponents of this research were members of the Berlin/Frankfurt school of Gestalt psychology, with whom Carnap was at least indirectly 
acquainted (Feest 2007). ${ }^{2}$ I therefore suggest that Carnap was aware of psychophysical research and that his proposal to translate protocol sentences into sentences about behavioural dispositions was in fact inspired by this tradition. ${ }^{3}$ With this claim I do not wish to refute the common assumption that the position advocated here Carnap is a kind of logical or analytical behaviourism. My thesis, rather, is that Carnap's articulation of this form of behaviourism (i.e., what Carnap called the "physicalizing" of qualitative terms), relied on a contingent empirical fact. And the way in which he imagined the empirical investigation of this fact was practiced by a particular (at the time quite dominant) approach within psychology. The implication I want to highlight here is that specific attempts to translate a psychological sentence ( $\mathrm{Mr} \mathrm{A}$ is angry) into physical sentence (Mr. A exhibits particular behaviors) are going to build on psychophysical research, which in turn will necessarily involve first-person data.

\section{Physical Language, Physicalized Observation Sentences and Psychology}

In his article "Psychology in a Physical Language" Carnap explored the implications of his physicalism for psychology as a science, specifically focusing on the question of the kinds of observation sentences were admissible in psychology. His targets were "observations of the mental states of others" (section 3) and "self-observations" (section 7).

With regard to observation-statements about the mental states of others (e.g., "Mr A is angry"), Carnap argued that insofar as such sentences are meaningful at all, they are only meaningful if they can be translated into statements about physical behaviours (about Mr. A's disposition to behave in certain ways). This allows for the derivation of sentences that state truth conditions for the sentence in question (about Mr. A's actual behaviour), though (as Carnap lays out) to treat them as such requires an additional premise, namely that in general people display the behaviors in question when angry. Carnap uses this to argue that if we want to test a particular sentence about the content of someone's mind, we have to (a) appeal to a general sentence about the kinds of physical conditions that need to be in place when we use the term "anger" and (b) point to a

\footnotetext{
${ }^{2}$ When discussing (both in his 1928 Aufbau and in his 1932 article about physical language) the question what are the units of experience that protocol language typically describes, he opted for those identified by Gestalt psychology.

${ }^{3}$ Even if this historical thesis does not hold up, I maintain that psychologists at the time would have recognized the similarity (and that's all that matters for my subsequent argument).
} 
particular set of physical conditions as instantiating the general conditions in question. Carnap then contrasts this ("rational") mode of justification with one where the emotional state of Mr. A is ascertained in a more "intuitive" way. He argues, however, that such intuitive sentences are either meaningless or can be translated into one that states the physical conditions that provide it with meaning. In section 7 , he picks up on this claim and says that the same is true of sentences about our own mental states: For example, when we utter a sentence like "I am nervous right now," this sentence is either meaningless or its meaning is provided by empirical truth conditions in a physical language (shaky hands, sweaty palms, etc.).

One might wonder whether (and if so, how) these considerations were relevant to the research practices of experimental psychology, as Carnap seems to suggest. In a nutshell, he had two answers to this: First, he claimed that by failing to appreciate his point about the semantics of psychological sentences, psychologists were prone to falling into a kind of psychophysical dualism (which is apparent, for example, when I say that I am in physical state $\mathrm{X}$ and in addition in mental state Y). Second, he cautioned against attributing a special kind of epistemic significance to first-person experiences (of other minds or of one's own mind), pointing out instead that in science one always deals with sentences about experiences, which should be treated as the behavioural outputs of complicated detection devices under particular physical conditions: "In principle, there is at most a gradual epistemic difference between the utterances of a fellow human being and a barometer" (p.124; translation by me). (see also p. 140 for similar statements).

Carnap repeatedly comments on the confused state of the then current psychology (for example "understanding" and "introspective" psychology), but he never quite says who is actually committing the above two errors. It is possible that he had in mind Wilhelm Dilthey, who had argued for understanding as a first-person mode of access to the subject matter of the human sciences. But Carnap's critique is a little confusing by virtue of the fact that Carnap also throws in a different psychological approach (again without mentioning any names), which studies "purposeful behaviour." I suggest that here Carnap had in mind the American neo-behaviorist Edward Chace Tolman, whom Carnap probably met at Vienna Circle meetings (or at least knew about via Egon Brunswick). ${ }^{4}$ Tolman emphasized the goal-directed nature of much behaviour and

\footnotetext{
${ }^{4}$ Tolman's book, Purposive Behavior in Animals and Men also appeared in 1932.
} 
essentially introduced proto-cognitive mental states to explain them. With respect to this explanatory practice, Carnap argues that it is acceptable as long as we realize that talk about purposes can be fully physicalized, i.e., translated into a language that specifies lawful regularities between specific stimuli and behavioural dispositions. It bears stressing that Dilthey's approach was quite different from Tolman's, insofar as the former talked about a mode of empirical access (understanding), whereas the latter talked about an explanatory concept (purpose). Hence, we can note that Carnap's critique of psychology was fairly broad.

While this does not come out in Carnap's article, one other likely target of Carnap's attack on psychology was the psychologist Karl Bühler, who was based in Vienna at the time. In 1927, just a few years before Carnap's "Psychology in a Physical Language", Bühler had published a book about what he had termed the "crisis of psychology" (Bühler 1927). In this book, Bühler specifically attacked the physicalism of Gestalt psychologists like Wertheimer, Köhler, and Koffka. According to Christoph Limbeck (2014), Carnap presented his ideas about a physicalist psychology in Bühler's colloquium on two separate occasions in the summer of 1930, where they gave rise to heated discussions. ${ }^{5}$ In the light of the hypothesis that Carnap's critique of contemporary psychology may have been directed at Bühler's 1927 book, and the light of the fact that Bühler, in this book, specifically opposed what he took to be the "physicalism" of the Berlin school of Gestalt psychology, it is not surprising that members of this school took an interest in Carnap's position.

\section{The Carnap/Duncker Exchange}

Carnap's 1932a article prompted a reply by Carl Duncker, a younger member of the Berlin school of Gestalt psychologists. This reply (and Carnap's subsequent response) demonstrates a deep mutual incomprehension. This is especially surprising in the light of my above thesis that Carnap's physicalism was informed by the psychophysical tradition, and that Gestalt psychological research has to be placed in that tradition. In this section, I provide a brief overview of the exchange, followed (in section 6) by an elaboration of my thesis.

\footnotetext{
${ }^{5}$ I would like to thank Christoph Limbeck for drawing my attention to this!
} 
Even though it is clear, especially at the beginning of his article, that Duncker had misunderstood some of Carnap's points, he did object to Carnap's characterization of the two supposed problems of psychology, namely the danger of falling into a mind-body dualism and the tendency to attribute too much epistemic significance to introspective data. Carnap had argued that these two problems could be avoided if one took the general stance of behaviorism: "The position advocated here is essentially in agreement with the psychology known as 'behaviorism'" (Carnap 1932a, 124, translation by me). ${ }^{6}$ Duncker argued that Gestalt psychological findings and methodology were more congenial to what Carnap was aiming at.

As mentioned above, Carnap claimed that his analysis of psychological sentences as translatable into physical ones could help psychologist avoid the inference that the two types of sentences referred to two separate kinds of "things." Duncker responded with utter incomprehension, stating that he was not aware of many contemporary psychologists who made substantial metaphysical assumptions about mind and body (Duncker 1932, 165), and that Gestalt psychologists were in fact physicalists, though he did not think that this had any implications for the goals or methods of non-behaviorist psychology (ibid. 176). He backed this up by claiming that psychophysics also viewed organisms as detection devices, like Canap, and that they endorse a principle of isomorphism between mental and physical processes (Duncker 1932, 174). However, Duncker also used some careless formulations, which seemingly contradicted his antimetaphysical stance. For example, he asserted that it was possible to conceptualize anger both as a behavioural disposition and as an inner experience. For Carnap these assertions showed Duncker to be falling in exactly the metaphysical trap that Carnap had warned about (Carnap 1932b, 186/7). Accordingly, Carnap responded with some surprise about Dunker's claim to be a physicalist, noting that he and Duncker clearly had in mind very different notions of physicalism: Carnap's physicalims was about the translatability of psychological language into physical language, whereas Duncker's physicalism was about finding the physical basis of introspectively accessible experience.

This brings us to Carnap's second critique of psychology, namely that of introspective methods. Carnap's rejection of introspection was closely related to his above-mentioned point about the dangers of being misled into a dualist metaphysical position. It was not aimed at the usage of this

\footnotetext{
${ }^{6}$ Here Carnap mentions that J. B. Watson's Der Behaviorismus had been translated into German in 1930.
} 
method as such, but at certain interpretations of its results, with its inherent danger of reifying the object of introspection, a point he reiterates in his reply to Duncker. In his response, Duncker stated that (a) Gestalt psychology (like all of psychophysics) relied on some kinds of self-reports since it was not clear how psychophysical laws could be formulated otherwise, but (b) that one did not have to be a behaviorist to reject the assumption that psychology aims at the "absolute content of a quale" (Duncker 1932, 167). Moreover, (c) when psychophysicists investigated (for example) color vision, they were not interested in the subjective experience of the color as such, but in their "order properties" (“Ordnungseigenschaften”). For example, if an individual experienced inverted qualia, this was irrelevant for the language of science, so long as that person responded to all the same stimuli in the same way as a person with 'normal' experiences. All of these points then lead up to Dunckers's somewhat exasperated question: "I ask once again, which ‘interpretation' of introspection is Carnap arguing against?’(Duncker 1932, 169).

Summing up, Duncker held that an endorsement of introspection was compatible with a lean metaphysics and with the notion of biological organisms as physical detection devices. Yet, at the same time, he held that introspection was an indispensable tool for the empirical investigation of such devices. Carnap, in his reply, repeated that he did not care what tools psychologists used, so long as the resulting sentences could be stated in a physical language. Thus, he took Duncker's conjectures to be irrelevant to his point.

\section{Some Underlying Issues}

In this section, I will argue that while it is true that Duncker misunderstood Carnap's point, it is also the case that Carnap was not receptive to the implications of Duncker's remarks, namely (a) that Carnap's physicalism did not have much practical relevance to at least some non-behaviorist psychology as it was being practiced at the time (or to the extent that it did, Carnap had failed to engage with the targets of his critique), and (b) that Carnap's physicalism was rooted in psychological research and hence he was well advised to engage with the foundations of that research. 


\subsection{The Heuristic Function of Introspection}

Clearly, one issue at play in the exchange between Darnap and Duncker was a version of the distinction between discovery and justification, with Carnap declaring scientific methodology irrelevant to the epistemological status of psychological claims such as "Mr A is angry." At that point in time, questions surrounding the production and epistemic status of first-person experiential reports had been debated within experimental psychology for well over half a century, following the work of Fechner, Brentano, G.E. Müller, and many others. The fact that Carnap simply ignored these debates must have seemed bizarre to a practicing scientist like Karl Duncker. Now, Carnap might have replied that those very discussions were themselves rooted in metaphysical assumptions, and that it was precisely for this reason that he was aiming at a purely formal analysis of scientific language rather than the metaphysical presuppositions of scientific practice. But the question is whether Carnap was entitled to this stance, since - as I argued above - his very conception of the physicalization of protocol sentence took for granted the project of psychophysics, that is, the project of discovering functional relationships between physicalist and mentalist descriptions. It is this fact that Duncker is also alluding to when arguing that even though experimental psychology mainly varies physical stimuli, this activity is heuristically guided by introspection (Duncker 173). This can be illustrated if we go back to Carnap's claim (see section 4 above) that the empirical conditions of application for a psychological sentence (such as "Mr. A is angry") were provided not only by an individual displaying the relevant behaviors, but also by an empirical law that described the types of behaviors typically displayed by angry people. Such laws, Carnap tells us, are the result of inductive generalizations. What Duncker is pointing out (on my construal) is that such generalizations are based on self-reports, and that therefore human subjectivity cannot be eliminated from the research process.

Again, Carnap might have replied that it was precisely the (merely) heuristic nature of introspection that rendered it irrelevant to serious logical analysis, and moreover, that even in the actual research (as he and Duncker agreed) only the introspective reports (not the introspective experiences themselves) counted. But even if we grant this, I would like to suggest that some of the unproductive harshness of this exchange could have been avoided if Carnap had acknowledged his debts to the psychophysical tradition. This might have helped him understand why Duncker was so irritated by Carnap's positive assessment of methodological behaviorism, since after all behaviorism, by virtue of not talking about mental states at all (physicalized or not) 
radically rejected the very method that Carnap's physicalism was built on, namely that of treating experiential reports as relevant scientific data. I will now argue that it might also have helped him provide a more nuanced description of the kinds of mind-body parallelisms available at the time.

\subsection{Varieties of Mind-Body Parallelisms}

As indicated above, Duncker rejected Carnap's claim that contemporary psychology's use of the material mode was leading it down the road to mind-brain dualism. But what did Duncker have in mind here?

Carnap's turn to the analysis of language was motivated by his aim to avoid metaphysics. While this specific program of antimetaphysics is particularly well known, the mid- to late $19^{\text {th }}$ century had seen a lot of debates about banning metaphysics from scientific and philosophical discourses. In this vein, already Fechner, in his 1860 Elements of Psychophysics, had formulated an account of the psychophysical relationship that aimed to steer clear of fruitless debates between materialists and idealists at the time. His response was to argue that mental and physical properties were token identical, but depending on one's perspective, one could only ever empirically apprehend one or the other and never both at the same time (Fechner 1860; Heidelberger 2004ab). While this type of position was often referred to as a kind of "parallelism" (see also Heidelberger 2003), a more apt description might be "dual-aspect theory," since this term does not suggest the existence of distinct substances or properties, but merely of distinct perspectives. It was precisely this notion that underwrote Fechner's empirical project of psychophysics. As Heidelberger (2003) explains, it is possible to distinguish between three layers of Fechner's parallelism: an empirical hypothesis about the functional relationship between physical and psychological descriptions, a dual-aspect theory of the mind/body relationship, and a cosmological thesis, according to which even inorganic processes have a mental side to them. I have argued above that Carnap not only shared Fechner's empirical hypothesis, but also his methodology of how to investigate this functional relationship (by varying stimuli and recording responses). While he clearly did not agree with Fechner's mind-body theory (let alone with his cosmological thesis), it bears stressing that Fechner's mind-body theory was not a kind of substance dualism. Rather, it was a dual aspect theory which scholars like Duncker may well 
have taken to lay the foundations for the very possibility of the psychophysical research that Carnap implicitly appealed to, when casting protocol sentences in terms of behavioural dispositions.

Now, it is clear that for Carnap, the linguistic description of behavioural dispositions (expressed in a physical language) merely ensured the meaningfulness of psychological sentences, whereas for Fechner they expressed psychophysical laws, i.e., laws that describe the empirical relationship between two types of the magnitudes: experiences and physical stimuli. But given that Carnap's semantic analysis also exploited (or at least assumed) the empirical relationship in question, it is well worth pointing out that Fechnerian psychophysics attempted to account for the existence of the empirical relationship without positing separate substances or even properties. By stating that the correspondence between the two languages was a crude empirical fact, Carnap may have been able to avoid metaphysical speculation, but there is also a sense in which this appeal is somewhat unsatisfactory. Moreover, it remains to me an open question to what extent Carnap's thinking about this was implicitly informed by some version of the dual-aspect theory that had underwritten Fechner's psychophysical research. Whether or not this was the case, I argue that for Duncker this may have been an intuitive way to think about the matter, which would account for his difficulties in comprehending Carnap's point.

\section{Conclusion}

I have argued that Carnap's account of protocol sentences (including those of psychology) was deeply informed by the psychophysical research tradition of the mid-19 $9^{\text {th }}$ to early $20^{\text {th }}$ centuries. In the light of this, I have provided an analysis of the exchange between Carnap and Duncker, in which Duncker questioned Carnap's contention that the methodological approach of behaviorism within psychology was congenial to his approach, arguing instead that Gestalt psychology came much closer to Carnap's outlook. I substantiated Duncker's assessment by providing a reading of Duncker's analysis that highlights the following two points: First, Gestalt-psychological research (and psychophysical research more generally), while giving a lot of weight to first-person experiential reports, did not necessarily invest them with epistemic certainty or treat them as being about irreducible qualia. Second, researchers in the psychophysical tradition (including 
Gestalt psychologists) were not necessarily committed to a mind-brain dualism, even if they aimed to formulate psycho-physical laws.

Given that Carnap's formal analysis relied on (or at least presupposed the possibility of) this research, I argue that it was unwise for Carnap to reject as irrelevant Duncker's points, both because it unnecessarily alienated a potential psychological ally and because it prevented Carnap from acknowledging the extent to which he and Duncker shared similar philosophical roots. It also prevented him from recognizing that his project of physicalizing protocol sentences (in the formal mode) relied on research that granted some epistemic authority to subjective experience (in the material mode).

In conclusion I argue that the way in which Carnap tried to insulate his philosophical project, as concerned with the "epistemological status" of psychological sentences, from the question of how such sentences are established was part of a general trend away from being concerned with scientific practice. While philosophy of psychology in the $19^{\text {th }}$ century had still been fairly practice-oriented (as evidenced, for example, in Fechner's 1860 Elements of Psychophysics or Brentano's 1874 Psychology from an Empirical Standpoint), the philosophical turn to formal analysis (along with the rise of behaviorism in the US), for some decades eclipsed the fact that much of psychology continued to make some use of first-person reports. As a result philosophers of psychology have only recently started to turn their renewed attention to questions about the meaning, role and justification of first-person reports in psychology's research practices. In this context, the physicalist analyses of introspection, as they were provided by advocates of Gestalt psychology, are still well worth considering. (Feest 2014)

\section{REFERENCES}

Bühler, K. (1927): Die Krise der Psychologie. Jena: Verlag Gustav Fischer.

Carnap. R. (1931a): Überwindung der Metaphysik durch logische Analyse der Sprache. Erkenntnis 2, 219-241

------ (1931b): Die physikalische Sprache als Universalsprache der Wissenschaft. Erkenntnis 2, $432-465$ 
(1932a): Psychologie in physikalischer Sprache. Erkenntnis 3, 107-142

(1932b): Erwiderung auf die vorstehenden Aufsätze von E. Zilsel und K. Duncker.

Erkenntnis 3, 177-188

---- (1963): The Physical Language as the Universal Language of Science. W. Alston \& G. Nakhnikian (Ed.): Readings in Twentieth-Century Philosophy. New York: The Free Press, pp. 393-424. (originally published as Carnap 1931b)

Duncker, K. (1932): Behaviorismus und Gestaltpsychologie (Kritische Bemerkungen zu Carnaps ,Psychologie in physikalischer Sprache'). Erkenntnis 3, 162-176

Feest, U. (2007): Science and Experience/Science of Experience: Gestalt Psychology and the Anti-Metaphysical Project of the Aufbau. Perspectives on Science 15(1), 38-62.

Feest, U. (2014): Phenomenal Experiences, First-Person Methods, and the Artificiality of Experimental Data. Philosophy of Science (in press)

Heidelberger, M. (2003): The Mind-Body Problem in the Origin of Logical Empiricism: Feigl and Psychophysical Parallelism. P. Parrini, M. Salmon, W. Salmon Logical Empiricism: Historical and Contemporary Perspectives, Pittsburgh, PA: Pittsburgh University Press

-----. (2004a): Fechner's (Wider) Conception of Psychophysics - Then and Now. Contribution to "Fechner Day 2004" XXth Meeting of the International Society for Psychophysics.

Coimbra, Portugal, 18-22 October, 2004 (unpublished)

-----. (2004b) Nature From Within: Gustav Theodor Fechner and His Psychophysical Worldview. Pittsburgh: University of Pittsburgh Press. (Translated from the German by C. Klohr)

Limbeck, Christoph (2014): Der Physikalismus bei Bühler und Carnap. (unpublished manuscript).

Neurath, O. (1932): Protokollsätze. Erkenntnis 3, 204-214 
Uebel, T. (2007): Empiricism at the Crossroads. The Vienna Circle's Protocol-Sentence Debate. Chicago: Open Court. 